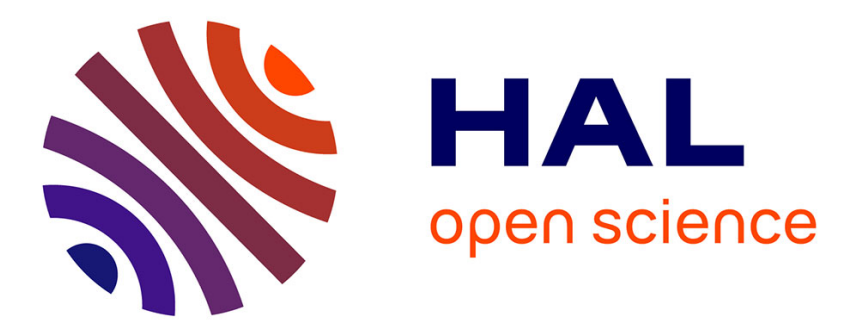

\title{
Angular Intermittency in QCD Jets
}

Philippe Brax, J.-L. Meunier, R. Peschanski

\section{To cite this version:}

Philippe Brax, J.-L. Meunier, R. Peschanski. Angular Intermittency in QCD Jets. Zeitschrift für Physik C Particles and Fields, 1994, 62, pp.649-658. hal-00163941

\section{HAL Id: hal-00163941 https://hal.science/hal-00163941}

Submitted on 19 Jul 2007

HAL is a multi-disciplinary open access archive for the deposit and dissemination of scientific research documents, whether they are published or not. The documents may come from teaching and research institutions in France or abroad, or from public or private research centers.
L'archive ouverte pluridisciplinaire HAL, est destinée au dépôt et à la diffusion de documents scientifiques de niveau recherche, publiés ou non, émanant des établissements d'enseignement et de recherche français ou étrangers, des laboratoires publics ou privés. 
Nice INLN $93 / 1$

January 1993

Saclay Spht/93-011

\title{
ANGULAR INTERMITTENCY IN QCD JETS
}

\author{
Ph. Brax\#, J.-L. Meunier*, and R. Peschanski\#
}

\begin{abstract}
Using two methods, via fluctuations and correlations, an analytical formula is derived for the factorial multiplicity moments in a QCD jet at the Double Leading Logarithm accuracy. The resulting self-similar dependence on the solid-angle cell size is characteristic of an intermittency behaviour in angular variables. The intermittency indices depend on the diffusion angle through the running of $\alpha_{s}$. Physical features of jet fluctuations such as collimation at large angles and saturation at small angles are well described in the perturbative framework. A parameter-free prediction of angular intermittency is proposed for $\mathcal{Z}^{0}$ decays into hadrons, assuming hadron-parton duality.
\end{abstract}

* Institut Non Linéaire de Nice, Université de Nice-Sophia Antipolis, Parc Valrose, 06108 Nice Cedex 2, France: Unité Mixte de Recherche du CNRS, UMR 129,

\# Service de Physique Théorique, Centre d'Etudes de Saclay, F-91191 Gif sur Yvette cedex, France: Laboratoire de la Direction des Sciences de la Matière du Commissariat à l'Energie Atomique. 


\section{Introduction}

The observation of multiplicity fluctuations in multiparticle production reactions at high energy is quite old [1], but it is only relatively recently ${ }^{[2]}$ that a quantitative method of analysis has been proposed and its results discussed in terms of the supposed reaction dynamics. Interestingly enough, a set of non trivial dynamical (by opposition to statistical) fluctuations has emerged, with the intriguing possibility ${ }^{[2,3]}$ of a self-similar scaling behaviour as a function of a small cell size in momentum space, called "intermittency" by analogy with hydrodynamics. However, the system at stake being ultrarelativistic and involving Quantum Physics, is very far from the classical areas where intermittent behaviour have been studied; Moreover, in Particle Physics, it is not easy to identify the possible origin of intermittency and more conventional models has been proposed as an alternative ${ }^{[4]}$. It is thus of importance to investigate this problem in the framework of the standard theory of strong interactions - Quantum Chromodynamics (QCD) - and in a case where it is known in principle to be calculable, namely in the weak-coupling perturbative regime of jet physics. This is the goal of the present paper.

The method designed to study multiplicity fluctuations from event to event in a given phase-space cell (to be defined later on) makes use of the so-called scaled factorial moments expressed as follows:

$$
\mathcal{F}_{q}(\Delta)=\frac{\langle n(n-1) \ldots(n-q+1)\rangle_{\Delta}}{\langle n\rangle_{\Delta}^{q}}, \quad \mathrm{I}-1
$$

where $n$ is the particle multiplicity in a phase space cell of size $\Delta$, in which multiplicity fluctuations are expected. Intermittency is characterized by a scale-invariant behaviour of the scaled factorial moments, namely

$$
\mathcal{F}_{q}(\Delta) \propto \Delta^{-f_{q}}
$$

where the constants $\left\{f_{q}\right\}$, usually called intermittency indices, play the role of anomalous dimensions of the fluctuation pattern. Indeed, the intermittency indices can in principle be related to the Renyi dimensions $\mathcal{D}_{q}$ characterizing a set of fluctuations. It reads ${ }^{[5]}$

$$
\begin{array}{ll}
\mathcal{D}_{q}=1-\frac{f_{q}}{q-1} & \mathrm{I}-3
\end{array}
$$


where $\mathcal{D}_{q}$ measures the defect of phase-space dimensionality due to intermittency (the dimension 1 in $[\mathrm{I}-3]$ corresponds to an uniform distribution in the $\Delta$ cells for all events).

It is to be noted that the same relations $[\mathrm{I}-1,2,3]$ can be quite equivalently interpreted and discussed in terms of correlations ${ }^{[6]}$. Indeed, on has the following relation:

$$
\mathcal{F}_{q}(\Delta) \propto \int \ldots \int_{\Delta} \prod_{1}^{q} d \omega_{i} \rho\left(\omega_{1}, \ldots, \omega_{q}\right)
$$

where $\rho$ is the differential multiplicity density distribution for $q$ particles in the phase space $\Delta$ integrated over the variables $\omega_{i}$. In particular, the existence of an intermittent behaviour [ $\mathrm{I}-2$ ], can be traced back to the existence of a singularity in $\rho$ at very short momentum distances ${ }^{[7]}$ and thus in the corresponding correlation functions ${ }^{[6]}$. In fact, these two different "languages", fluctuations and correlations, though equivalent, lead to two different calculation methods of the factorial moments in the perturbative QCD framework, as we shall see further on. This will serve as a non trivial check of the calculations, as the final result is obtained in both ways.

A behaviour compatible with [ I - 2] has been found in various reactions analyzed in terms of the rapidity $y$, azimuth angle $\varphi$ and/or transverse momentum $P_{\perp}$ of the produced particles around the jet reference axis ${ }^{[8]}$, giving rise to a lot of phenomenological discussions and models of various types ${ }^{[9]}$. However, the observation of an intermittent behaviour in $\mathrm{e}^{+}-\mathrm{e}^{-}$anihilation into hadrons ${ }^{[10]}$ and especially in the $\mathcal{Z}^{0}$ hadronic decays at $\operatorname{LEP}^{[11]}$ has raised a particular interest. Beside the quality of high-precision, high-statistic events allowing the non-trivial extraction of the factorial moments from data, it offers the possibility of a comparison with perturbative QCD predictions, either "indirectly" through Monte Carlo simulations including full hadronisation models or "directly", at the level of partonic shower and assuming parton-hadron duality ${ }^{[12]}$.

Indeed, the evidence that Monte-Carlo models of $\mathrm{e}^{+}-\mathrm{e}^{-}$annihilation into jets could naturally lead to intermittent patterns has been numerically obtained in Ref.[12], but the hadronization scales imply a "saturation effect", namely a stabilisation of factorial moments beyond some small cell-size in rapidity. As a matter of fact at LEP energy, Monte-Carlo models based on Perturbative QCD plus an hadronization model give a satisfactory description ${ }^{[11]}$ of 1,2 and 3 -dimensional factorial moments, with some non-negligeable, but limited discrepancies when various experimental cuts are performed. However, here also, the role of hadronization in the final result can be important, or at least remains ambiguous. Indeed, two different Monte Carlo's procedures, the "Parton Shower Models" and the "Matrix Element Models" give quite similar results ${ }^{[11]}$, while the former admits a rather extended parton cascading and the second is limited to at most 4 final partons before hadronisation takes place. In this case, almost all the effect is encoded in the hadronization model.

On the theoretical side of the question, it is possible to estimate factorial 
moments at the parton level using the leading log approximation of the perturbative expansion. For instance, evidence for an intermittent behaviour was numerically obtained from a simulation of a gluon cascade ${ }^{[13]}$, using a simplified version of a QCD jet. The results can be compared with data by assuming the $\mathrm{LPHD}^{[14]}$ (local parton-hadron duality hypothesis). Then, a quite systematic study has been analytically performed in the framework of the so-called "dipole approximation" of the quark-gluon radiation allowing a determination ${ }^{[15]}$ of fractal dimensions related to gluon cascading. Note that these dimensions are considered in Ref. [15] as being different from intermittency anomalous dimensions, we will later come back to this interesting discussion. Two more recent papers, one using planar diagrams ${ }^{[16]}$, and one in the framework of multiparton correlations ${ }^{[17]}$ will also be discussed in comparison with our methods and results.

Our aim is to obtain the most general Perturbative QCD prediction at the double leading log approximation (DLLA) for the pattern of fluctuations/correlations within a parton jet. In the analysis based on fluctuations, one will be inspired by some methods used in the paper of ref. [16]. For the correlation analysis, the method is borrowed from the calculation of energy-multiplicity correlators, which already led Y. Dokshitzer and S. Troyan to the discussion of factorial moments ${ }^{[18]}$ and have been recently developped in a different context, see ${ }^{[19]}$.

The key points we want to address, using the present state of the art ${ }^{[20]}$, can be summarized in a series of questions:

i) Is Perturbative QCD intrinsically intermittent?

ii) In which variables this property of fluctuations shows up behaviour?

iii) What are the anomalous dimensions characterizing the intermittency

iv) How to perform the phenomenological analysis of the DLLA predictions?

v) Why intermittency appears in Perturbative QCD calculations?

As we shall now see in the derivation, the main result of our study is that the question $i$ ) is investigated in detail and answered positively; From that study, one also gets a precise answer to questions $i i)-v$ ). This is the subject of the present paper, which is organized in the following way: In section II, we use a first method - the fluctuation approach - to get a first hint to the calculation of the factorial moments and the emergence of angular variables in the structuration of fluctuations. However, an ambiguity remains about the energy variable, which in section III is resolved by the method of correlations while confirming the validity of the first results. The fourth section is devoted to the description of the resulting intermittent behaviour and to a discussion of the important difference between fixed and running coupling constant which leads to QCD scaling violation effects of a new nature ("saturation effects"). The resulting "angular intermittency" property is discussed both for its phenomenological implications and its comparison with previous approaches. Finally, in section V, we propose some comments on the roots of the intermittent behaviour in a quantum field theory like QCD and discuss open 
problems and suggestions for further study.

\section{Intermittency from fluctuations in a QCD jet}

Let us start from the well-known multiplicity generating function ${ }^{[20]}$ of one gluon jet with opening angle $\Theta_{0}$ and energy $E, \mathcal{Z}\left(E \Theta_{0}, v\right)$ :

$$
\begin{aligned}
& \mathcal{Z}\left(E \Theta_{0}, 0\right)=1 \\
& \left.\frac{\partial \mathcal{Z}}{\partial v}\right|_{v=0}=\langle n\rangle=\mathbb{F}_{1} \\
& \left.\frac{\partial^{q} \mathcal{Z}}{\partial v^{q}}\right|_{v=0}=\langle n(n-1)(\ldots)(n-q+1)\rangle=\mathbb{F}_{q}
\end{aligned}
$$

where $\mathbb{F}_{q}$, by difference with [I - 1], are for non-normalized and global multiplicity moments. Note that the generating function depends kinematically on the product $E \Theta_{0}$, i.e. the highest parton transverse momentum ${ }^{[20]}$.

$\mathcal{Z}\left(E \Theta_{0}, v\right)$ obeys the following evolution equation ${ }^{[21]}$ :

$$
\frac{\partial \mathcal{Z}}{\partial \ln \Theta_{0}} \equiv \dot{\mathcal{Z}}=\frac{1}{2} \int_{z_{0}}^{1} \frac{\alpha_{s}}{2 \pi} \Phi_{A}(z)\left[\mathcal{Z}\left(E z \Theta_{0}, v\right) \mathcal{Z}\left(E(1-z) \Theta_{0}, v\right)-\mathcal{Z}\left(E \Theta_{0}, v\right)\right] d z
$$

where $\Phi_{A}(z)=4 C_{A}\left(\frac{1}{z}+\frac{1}{1-z}+\ldots\right) C_{A}$ is the gluon color factor, and $z_{0}=\mu / E \Theta_{0}$ the cut-off from the non-perturbative region. This equation is given in terms of an energy integral and seems a priori different from the multiplicity density integral typical of an intermittent Random Fragmentation Model ${ }^{[22]}$ whose evolution is however also governed by a quadratic non-linear equation (see farther, Eq.[ II - 8,9 ]). Up to non leading terms the equation [II - 2] can be simplified to :

$$
\dot{\mathcal{Z}} \simeq \mathcal{Z} \int_{z_{0}}^{1} \gamma_{0}^{2} \frac{d z}{z}\left[\mathcal{Z}\left(E z \Theta_{0}, v\right)-1\right] \quad \text { II }-3
$$

with $\gamma_{0}^{2}=4 C_{A} \alpha_{s} / 2 \pi$.

Let us first consider the simplified case of a fixed coupling constant $\alpha_{s}$. The first-moment equation can be integrated from the smallest angle possible, $\Theta_{\mu}=$ $\mu / E$, (where $\mu$ is some infrared cut-off of the order of the hadronic scale), up to $\Theta_{0}$ :

$$
\langle n\rangle \equiv \mathbb{F}_{1} \propto \exp \left[\gamma_{0} \ln \frac{E \Theta_{0}}{\mu}\right]
$$

which represents the well-known total multiplicity of gluons in a jet of angular aperture $\Theta_{0}$ in the fixed-coupling regime. Then, the other moments equations can be obtained from [II -3$]$ as :

$$
\dot{\mathbb{F}}_{q}=\sum_{1}^{q} C_{q}^{p} \mathbb{F}_{q-p} \int_{z_{0}}^{\infty} \frac{d z}{z} \gamma_{0}^{2} \mathbb{F}_{p}\left(z E, \Theta_{0}\right) \quad \text { II }-5
$$


where the $C_{q}^{p}$ are the binomial coefficients. As is well known ${ }^{[20]}$ such distributions follow asymptotically the KNO scaling law, namely

$$
\begin{array}{ll}
\mathbb{F}_{p}=c_{p} \mathbb{F}_{1}^{p} & \text { II }-6
\end{array}
$$

where $c_{p}$ are known constants. Using [ II -4$]$ and [ II -6 ], the integral in [ II -5 ] can be done explicitely, and the equations takes the following form:

$$
\dot{\mathbb{F}}_{q}=\sum_{1}^{q} C_{q}^{p} \mathbb{F}_{p} \mathbb{F}_{q-p} \frac{\gamma_{0}}{p} \quad \text { II }-7
$$

where one can notice the transformation of the convolution in [ II - 5] into a mere product, independent of the values of the constants $c_{p}$.

As a matter of fact, this set of equations is identical to the one obtained for a generating function of the global multiplicity distribution $\mathcal{H}(\Theta, u)$ of a semi-random fragmentation model ${ }^{[22-23]}$ (Figure 1) which obeys the generic equation:

$$
\begin{array}{ll}
\dot{\mathcal{H}}=\mathcal{H}(\widetilde{\mathcal{H}}-1) & \text { II }-8
\end{array}
$$

with

$$
\widetilde{\mathcal{H}}(u)=\int_{0}^{1} r(\mathrm{w}) \mathcal{H}(u \mathrm{w}) d \mathrm{w} \quad \text { II }-9
$$

whose kernel is given by the distribution:

$$
r(\mathrm{w})=\delta(\mathrm{w})+\left.\frac{\gamma_{0}}{\mathrm{w}}\right|_{+}
$$

where the + stands for the Principal-Value distribution. The equivalence between [ II -7$]$ and [ II -8 ] is easily obtained by differentiation in $u$ at $u=0$.

More precisely, the equations [II - 3], [II - 9] are obtained in the framework of Random-Branching Random-Cascading fragmentation models ${ }^{[22-23]}$, with a "time" variable to be identified, up to a constant, with $\ln \Theta_{0}^{-1}$. The model can be described as a tree of random multiplicative weights $\mathrm{w}$ (Figure 1), with the following recepe: There is a unit probability per unit time to have a new branching; At each such vertex, one branch brings the (deterministic) weight 1 and the other gives a random weight $\mathrm{w}$ following the probability law $r(\mathrm{w})$ defined by [II -9$]$. In ${ }^{[22]}$, it has been called a semi-random model. The $q \neq 0$ moments of the distribution $r$ are precisely $\overline{\mathrm{w}^{\mathrm{q}}}=\gamma_{0} / q$, while its normalization is kept to be 1 . This kind of kernel has been used in another context ${ }^{[2]}$ and exhibits explicit intermittency properties. For this class of fragmentation models to be compared with QCD cascading, the corresponding (positive) time variable runs from 0 to $\ln E \Theta_{0} / \mu$.

Now comes the step between the global formulation of a random cascading model (formula [ II - 7] for the multiplicity moments $\mathbb{F}_{q}$ and [ II - 8] for their 


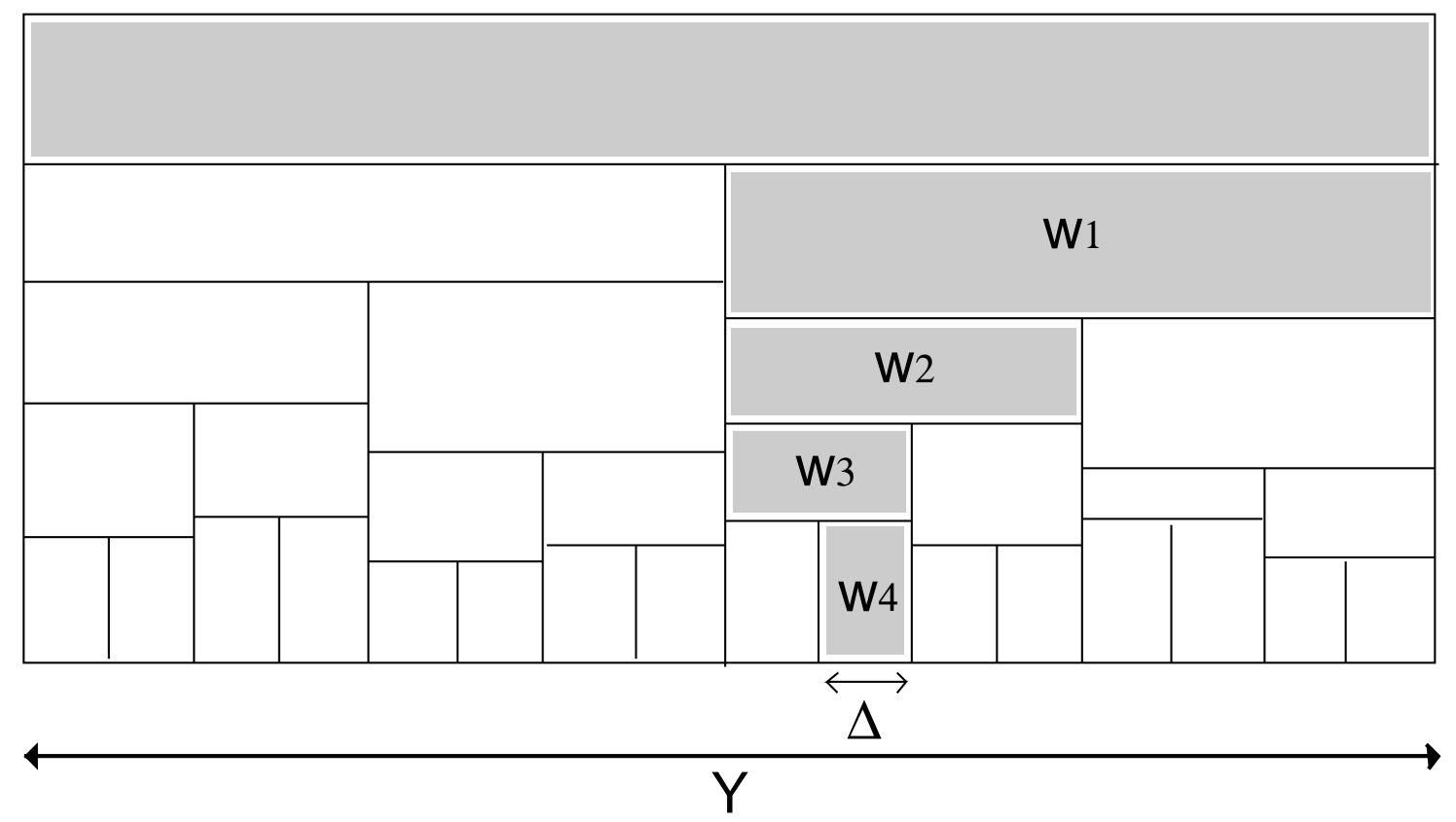

Figure 1

Figure 1 : Sketch of the random fragmentation cascade. In grey, the "history" of the bin $\Delta$, i.e. the successive random multiplicative factors $\mathrm{w}$ which define the multiplicity density in $\Delta$ for one event. $Y$ (resp. $\Delta$ ) is the initial (resp. final) observation range. As a simple illustration from the figure, the bin density is $\rho_{\Delta}=\rho_{Y} \mathrm{w}_{1} \mathrm{w}_{2} \mathrm{w}_{3} \mathrm{w}_{4}$ where $\rho_{Y}$ is the initial density, and the w's are the random or unity weights (see text) corresponding to the semi-random structure.

generating functions $\mathcal{H}$ ) to a local formulation for the factorial moments $\mathcal{F}_{q}$ defined in $[\mathrm{I}-1]$. For this sake, one used to introduce the generating fonction of the local moments, namely $H_{\Delta}$.

In the random fragmentation models described in ref. [22-23], the development of the cascade gives rise to the following equation governing the local intermittent fluctuations:

$$
\dot{H}_{\Delta}=\widetilde{H}_{\Delta}-H_{\Delta},
$$

where $H_{\Delta}$ is thus the generating function of the multiplicity distribution in a cell of size $\Delta$. Qualitatively, Equation [ II - 11] comes from the fact that along the path leading to the cell $\Delta$, see Figure 1, one encounters either weights 1 or w, taking into account the semi-random structure of the model. The technical derivation requires some care and has been obtained in two ways, either from the global equation ${ }^{[22]}$ or from a subdimensional equation ${ }^{[23]}$. Note that in order to figure out the (average) 
number of cascading steps, the size of the cell $\Delta$ is to be compared with the large scale of the problem, namely the initial range $Y$ (this model was originally used for rapidity cells ${ }^{[2,23]}$, but it can be extended to any other relevant variable). The corresponding "time" variable is indeed related to $t=\log Y / \Delta$.

Let us now come back to QCD. Indeed, in the present case, the cell one is led to consider is angular, and its size $\Theta$ has to be compared with the only large scale one has in the problem (on an event by event basis), namely the observation angle $\Theta_{0}$. Thus, the natural evolution variable involved in the equation [ II -11 ] is $t=\log \Theta_{0} / \Theta$. This choice can also be understood as a reflexion of the QCD angular ordering ${ }^{[20]}$; Angular ordering is the property of QCD jets at leading-log order that, when partons are detected in the direction $\Theta_{0}$ with respect to a jet axis, the maximal aperture of the "sub-jet" of partons contributing to the observation is of the order of the observation angle, with a subsequent degradation of the diffusion angles along the cascade.

From [II - 11], one obtains the following solution for the angular factorial moments :

$$
\begin{aligned}
\mathcal{F}_{q}\left(\Theta_{0}, \Theta\right) & \propto\left[\frac{\Theta_{0}}{\Theta}\right]^{(q-1)\left(1-\mathcal{D}_{q}\right)}, \text { with } \\
\mathcal{D}_{q} & =\gamma_{0} \frac{q+1}{q}
\end{aligned}
$$

expressing an exact power law $[\mathrm{I}-2]$ in the angular variable $\Theta$, for a fixed coupling constant, i.e. fixed $\gamma_{0}$.

A series of remarks can be made about the result [ II - 12].

i) The fixed coupling constant regime

The intermittency property [ II - 12] is exactly realized at fixed coupling constant. However, one has to verify the condition $\mathcal{D}_{q}<1$. or, in words, that the Renyi dimension is smaller than the support dimension of the set of fluctuations ${ }^{[5,3]}$. In [II -12$]$ this is obtained for $\gamma_{0} \leq \frac{2}{3},(q \geq 2)$, or equivalently, $\alpha_{s} / 2 \pi \leq 1 / 9 N_{c}$, which means a rather weak coupling regime. This corresponds to the QCD parton cascade in its initial development. A correct treatment of the full cascade requires taking into account the running QCD coupling.

\section{ii) 2-dimensional result and collimation}

Motivated by the appearance of an angular variable in [ II -12$]$, one can introduce the azimuthal angle $\Phi$ in the game by considering the jet development in the overall solid angle specified by the couple $(\Theta, \Phi)$. Indeed, a slight modification of the arguments developped for [II - 11] shows that one can embed a two-dimensional branching in a higher $d$-dimensional phase space; It is enough (with some conditions of uniformity $\left.{ }^{[24]}\right)$ to introduce $(d-1)$ other branches at the same vertex with zero multiplicative weight (no particle produced), in the definition of the random cascading model. This can be interpreted as the collimation property of QCD jets. Indeed, it is not too difficult to realize that the global QCD equation [II - 3] remains unchanged by integration over the $d-1$ other variables $(\Phi$, in our case) while the 
local equation [ II - 11] becomes :

$$
\dot{H}_{\Delta}=\widetilde{H}_{\Delta}-d H_{\Delta}+d-1
$$

With this physical interpretation and using [ II - 13], one finds for the embedding into the 2-dimensional space $(\Theta, \Phi)$, i.e for the factorial moments in a solid angle phase-space cell of size $\Delta \Omega \simeq \pi \Theta^{2}$,

$$
\begin{aligned}
\mathcal{F}_{q}(\Delta \Omega) & \propto\left[\frac{\Theta_{0}}{\Theta}\right]^{2(q-1)\left(1-\mathcal{D}_{q}\right)}, \text { with } \\
\mathcal{D}_{q} & =\frac{\gamma_{0}}{2} \frac{q+1}{q}
\end{aligned}
$$

Note that the energy-momentum development of the QCD cascade corresponding to formula [II - 14] implies a "2/3-collimation" at each branching, since $1 / 3$ of the cone contains no particles in the equivalent fragmentation-model framework.

\section{iii) The running coupling constant case}

The extension of the fluctuation analysis to the QCD running coupling constant is not too difficult. The whole derivation essentially remains the same, except that one has to take into account the $\alpha_{s}$ dependence on $\Theta$ in the solution of the differential equations $[$ II -3$]$ to $[$ II -11$]$. The final formula reads:

$$
\begin{aligned}
\mathcal{F}_{q}\left(\Theta_{0}, \Theta\right) \propto\left[\frac{\Theta_{0}}{\Theta}\right]^{d(q-1)\left(1-\mathcal{D}_{q}\right)}, \text { with } \\
\mathcal{D}_{q}\left(\Theta, \Theta_{0}\right)=\frac{q+1}{d q} \frac{\int_{\Theta}^{\Theta_{0}} \gamma_{0}\left(\alpha_{s}(\theta)\right) d \theta / \theta}{\ln \Theta_{0} / \Theta}
\end{aligned}
$$

One retrieves expression [ II - 12] by the approximation $\alpha_{s}=$ cst. As can be remarked from formula [ II - 15], the $\ln \Theta$ variation of the coupling constant will induce a modification of the behaviour of $\mathcal{D}_{q}$ which will, in general, depends on both $\Theta_{0}$ and $\Theta$ and not on their only ratio. Thus one may expect a modification of the power-law [II - 14] - and of the intermittent behaviour [I - 2] - due to the running of the coupling constant. This effect is in fact related to the well-known QCD scaling violation of the structure functions observed in deep inelastic scattering. It is thus of importance to take into account the correct $\alpha_{s}(\Theta)$ behaviour to compute the Renyi dimensions $\mathcal{D}_{q}$ in $[$ II -15$]$. However, this behaviour depends on the genuine scale on which $\alpha_{s}$ depends. A good candidate ${ }^{[21]}$ is the parton relative transverse momentum $P_{\perp}$, but then, care must be taken of the evolution of the parton energy $k$ in the jet, since $P_{\perp} \simeq k \Theta$. Neglecting the energy loss would allow us to choose $k \equiv E$ (the initial energy of the gluon jet), another extreme being to consider $k \equiv \mu$, the infra-red cut-off; We shall complete the discussion in the next section III, removing this ambiguity, and shall define the concept of angular intermittency implied by [ II - 15]. 


\section{Intermittency from correlations in a QCD jet}

Let us now introduce the method of refs. ${ }^{[18,19]}$. For sake of simplicity, we start again considering the fixed coupling regime. The correlation of two multiplicity flows in this case is sketched in the graphs of Fig.2a-b. Here the gluon jet with initial energy $E$ and production angle $\Theta_{0}$ evolves producing a soft offspring $k$ which then splits into two partons (gluons) with relative angle $\Theta_{12} \leq \Theta \ll \Theta_{0}$ generating the registered particle flows. Due to angular ordering the resulting 2-body correlation can be written (after a few thechnical manipulations described farther) as a convolution of the energy spectrum of parton $k$ with the product of the two multiplicity factors, namely :

$$
(4 \pi)^{2}\left(1-\cos \Theta_{12}\right) \frac{d^{2} N^{(2)}}{d \Omega_{1} d \Omega_{2}}=4 C_{F} \frac{\alpha_{s}}{2 \pi} \int^{E} \frac{d k}{k} \tilde{D}\left(\frac{E}{k}, \frac{\Theta_{0}}{\Theta}\right) \cdot 2 \dot{N}\left(k \Theta_{12}\right) N\left(k \Theta_{12}\right)
$$

where $\tilde{D}$ denotes the energy spectrum originating from cascades with parton emission angles larger than the given $\Theta$ and $N$ means the multiplicity derivative with respect to the argument. Notice that the colour factor $C_{F}$ in the emission probability of the gluon $E$ corresponds to the case of the quark as an original parton shown by a horizontal line in Figs.2. This quark line can be thought of as determining the main direction of the hard process under consideration (e.g., the quark jet direction in $e^{+} e^{-}$annihilation). Note that Eq.[ III -1 ] is written for the differential distribution as a function of the solid angles $\Omega_{1}$ and $\Omega_{2}$, with $\Theta_{12}$ the angle between the two triggered partons.

The last factor in [III - 1] has emerged after performing the integration over the relative energy fraction, $z$, in the decay of the parton $k$,

$$
\int_{0}^{1} d z 4 C_{A} \frac{\alpha_{s}}{2 \pi}\left\{\frac{1}{z}+\frac{1}{1-z}+\ldots\right\} N\left(z k \Theta_{12}\right) N\left((1-z) k \Theta_{12}\right) \approx 2 \dot{N}\left(k \Theta_{12}\right) N\left(k \Theta_{12}\right)
$$

where we only accounted for the infrared-singular (logarithmic) terms of the gluon splitting probability and made use of the DLLA evolution equation for the (gluon) jet multiplicity ${ }^{[20]}$,

$$
\dot{N}(Q) \equiv \frac{\partial}{\partial \ln Q} N(Q)=\int_{0}^{1} \frac{d z}{z} 4 C_{A} \frac{\alpha_{s}}{2 \pi} N(z Q) \quad \text { III }-3
$$

To obtain the factorial moment $F_{2}$, i.e. the integral multiplicity correlator, one has to integrate [III -1 ] over $d \Omega_{1} d \Omega_{2}$ keeping the relative angle between the two directions smaller than $\Theta$. Observing that

$$
\int \frac{d \Omega_{1} d \Omega_{2}}{(4 \pi)^{2}} \frac{\vartheta\left(\Theta-\Theta_{12}\right)}{\left(1-\cos \Theta_{12}\right)}=\frac{d \Omega_{1}}{4 \pi} \int_{0}^{2 \pi} \frac{d \phi_{12}}{2 \pi} \int_{0}^{\Theta} \frac{\sin \Theta_{12} d \Theta_{12}}{2\left(1-\cos \Theta_{12}\right)} \approx \frac{d \Omega_{1}}{4 \pi} \int_{0}^{\Theta} \frac{d \Theta_{12}}{\Theta_{12}}
$$




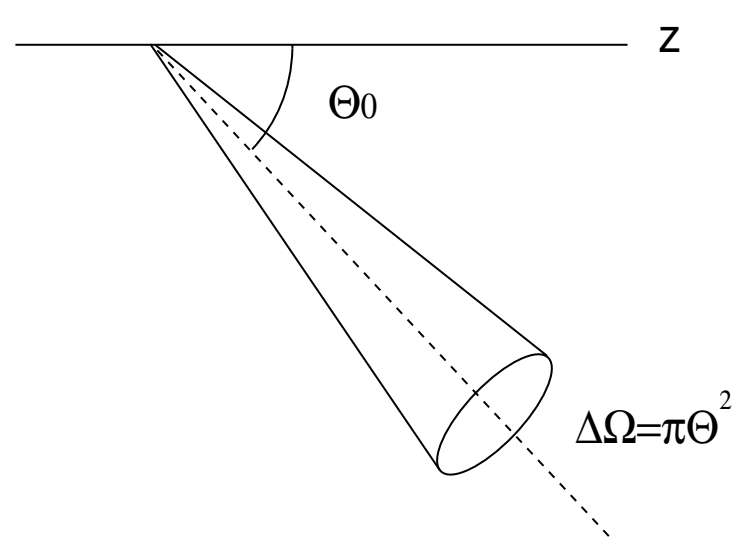

Figure 2a

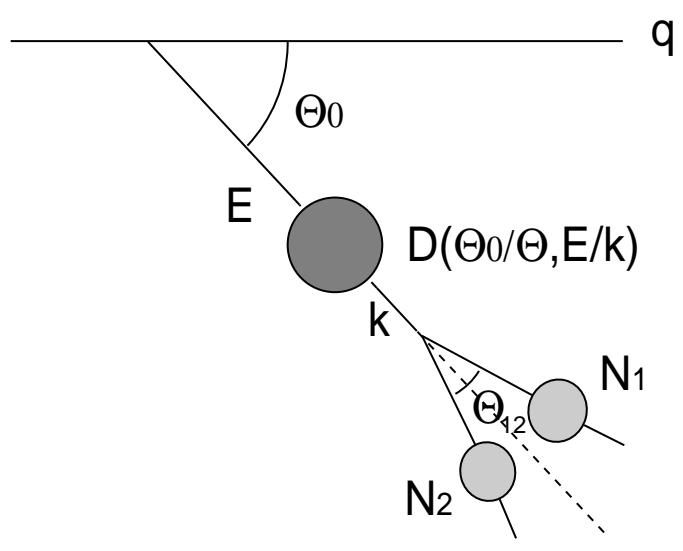

Figure $2 b$

Figure 2 : Kinematics of parton-parton QCD correlations; a) Location of the phase-space cell $\Delta$ in polar coordinates; b) schematic representation of the convolution formulae [ III - 1,5].

we obtain the formula :

$$
(4 \pi) \frac{\Delta N^{(2)}}{\Delta \Omega}=\frac{4 C_{f} \alpha_{s}}{2 \pi} \int^{E} \frac{d k}{k} \tilde{D}\left(\frac{E}{k}, \frac{\Theta_{0}}{\Theta}\right) \cdot N^{2}(k \Theta) \quad \text { III }-5
$$

where $\Delta \Omega$ stands for the (small) solid angle size of the phase space cell in consideration (see Fig. 2b).

The evolution equation for the distribution $\tilde{D}$,

$$
\tilde{D}\left(\frac{E}{k}, \frac{\Theta_{0}}{\Theta}\right)=\delta(\ln E / k)+\int_{\Theta}^{\Theta_{0}} \frac{d \Theta^{\prime}}{\Theta^{\prime}} \int_{k}^{E} \frac{d \ell}{\ell} 4 C_{A} \frac{\alpha_{s}}{2 \pi} \tilde{D}\left(\frac{E}{\ell}, \frac{\Theta_{0}}{\Theta^{\prime}}\right), \quad \text { III }-6
$$

can be solved by means of a Mellin transform in the $k / E$ variable which gives :

$$
\tilde{D}_{n}\left(\frac{\Theta_{0}}{\Theta}\right)=\exp \left[-\frac{\gamma_{0}^{2}}{n} \ln \left(\frac{\Theta}{\Theta_{0}}\right)\right] \quad \text { III }-7
$$

where

$$
\tilde{D}_{n}\left(\frac{\Theta_{0}}{\Theta}\right)=\int_{0}^{1} x^{(n-1)} \tilde{D}\left(\frac{1}{x}, \frac{\Theta_{0}}{\Theta}\right) d x, x \equiv k / E
$$

Using now the stationnary phase argument in the inverse Mellin transform one obtains:

$$
\tilde{D}\left(\frac{E}{k}, \frac{\Theta_{0}}{\Theta}\right) \simeq \exp \left[2 \gamma_{0} \sqrt{\ln \frac{E}{k} \ln \frac{\Theta_{0}}{\Theta}}\right] \quad \text { III }-8
$$


$\tilde{D}$ monotonically increases while $k$ decreases down to the value $\mu / \Theta$ where $\mu$ denotes again the infra-red cutoff. Starting from [III -5$]$, we need to convolute the $\tilde{D}$ distribution with the square of the multiplicity factor .

More generally, when not only two but $q>2$ particles are registered in the final state within the small cell $\Theta$, one has to consider the $q^{t h}$ inclusive multiplicity correlator. Using similar arguments, related to the DLLA properties of Perturbative QCD for a jet ${ }^{[18]}$, one is led to weight $\tilde{D}$ with the $q^{t h}$ power of the mean multiplicity. This gives :

$$
(4 \pi) \frac{\Delta N^{(q)}}{\Delta \Omega}=\frac{4 C_{f} \alpha_{s}}{2 \pi} \int^{E} \frac{d k}{k} \int \frac{d n}{2 i \pi} \exp [S(n, k, \Theta)] \quad \text { III }-9
$$

with

$$
S(n, k, \Theta)=n \ln \left(\frac{E}{k}\right)+\frac{\gamma_{0}^{2}}{n} \ln \left(\frac{\Theta_{0}}{\Theta}\right)+q \gamma_{0} \ln \left(\frac{k \Theta}{\mu}\right) \quad \text { III }-10
$$

where the introduction of the infra-red cut-off $\mu$ comes with the multiplicity, see [II -4$]$. We can now make use of the steepest descent technics in $k$ together with the stationnary phase space approximation in $n$ and get the result:

$$
\begin{aligned}
(4 \pi) \frac{\Delta N^{(q)}}{\Delta \Omega} & \propto\left(\frac{E \Theta}{\mu}\right)^{q \gamma_{0}}\left(\frac{\Theta_{0}}{\Theta}\right)^{\frac{\gamma_{0}}{q}}, \text { with } \\
\ln \frac{E}{k} & =\frac{1}{q^{2}} \ln \frac{\Theta_{0}}{\Theta}
\end{aligned}
$$

Note that, in this case of a fixed coupling constant, there may be a sizeable difference between the hierarchy in angles and the one in energy, at least for small-rank correlations. The result [III -10$]$ is valid if one remains in the perturbative regime i.e. $\ln \left(\frac{k \Theta}{\mu}\right)>0$. Defining the rescaled variable $x_{\mu}$ as:

$$
x_{\mu}=\frac{\ln \left(\frac{\Theta_{0}}{\Theta}\right)}{\ln \left(\frac{E \Theta_{0}}{\mu}\right)}
$$

where $\mu / E$ is the minimal angle $\Theta$ one may safely consider, the previous condition reads:

$$
x_{\mu}<\frac{q^{2}}{q^{2}+1},\left(=\frac{4}{5} \text { when } q=2 \text { and } \simeq 1 \text { for } q \text { large }\right) .
$$

$x_{\mu}>\frac{4}{5}$ is thus the correct limit of the perturbative regime. 
The scaled moments can now be calculated, and using $\Delta \Omega \simeq \pi \Theta^{2}$, one retrieves formula [ II - 14] under the guise:

$$
\begin{aligned}
\mathcal{F}_{q}(\Delta \Omega) & \propto\left[\frac{\Theta_{0}^{2}}{\Theta^{2}}\right]^{(q-1)\left(1-\mathcal{D}_{q}\right)}, \text { with } \\
\mathcal{D}_{q} & =\frac{\gamma_{0}}{2} \frac{q+1}{q}
\end{aligned}
$$

and, by integration on the azimuth, formula II- 12 .

Let us come back now to the perturbative QCD calculation with the appropriate running coupling constant $\alpha_{s}\left(P_{\perp}=k \Theta\right)$. To estimate the typical momenta $k$ in the main convolution formula [ III - 1] we use again the Mellin transform, as in formulae [III $-9,-10$ ], but in the context of a running $\alpha_{s}$, one gets :

$$
\frac{\Delta N^{(q)}}{\Delta \Omega} \propto \int \frac{d k}{k} \int \frac{d \omega}{2 \pi i} \exp [\mathcal{S}(\omega, k, \Theta)] \quad \text { III }-14
$$

with

$$
\mathcal{S}(\omega, k, \Theta)=\omega \ln \frac{E}{k}+\int_{k \Theta}^{E \Theta_{0}} \frac{d t}{t} \gamma_{\omega}\left(\alpha_{s}(t)\right)+q \cdot \int_{\mu}^{k \Theta} \frac{d t}{t} \gamma_{0}\left(\alpha_{s}(t)\right) \quad \text { III }-15
$$

and

$$
\gamma_{\omega}\left(\alpha_{s}\right)=\frac{1}{2}\left[-\omega+\sqrt{\omega^{2}+4 \gamma_{0}^{2}}\right]
$$

where $\gamma_{\omega}$ is the standard DLA anomalous dimension. Then we evaluate [III-14] by steepest descent in $k$ and stationary phase in $\omega$ to get

$$
\begin{gathered}
-\frac{\partial}{\partial \ln k} \mathcal{S}=\omega+\gamma_{\omega}\left(\alpha_{s}(k \Theta)\right)-q \gamma_{0}\left(\alpha_{s}(k \Theta)\right)=0 \\
\frac{\partial}{\partial \omega} \mathcal{S}=\ln \frac{E}{k}+\int_{k \Theta}^{E \Theta_{0}} \frac{d t}{t} \frac{\partial}{\partial \omega} \gamma_{\omega}\left(\alpha_{s}(t)\right)=0
\end{gathered}
$$

Making use of [III - 15] we find the following solution for the preferred value $\langle\omega\rangle$ :

$$
\langle\omega\rangle=\frac{q^{2}-1}{q} \gamma_{0}\left(\alpha_{s}(k \Theta)\right)
$$

and substitute it into the second relation [ III - 16] to obtain the equation which determines implicitly $\langle k\rangle$ :

$$
\ln \frac{E}{k}=\ln \frac{\Theta_{0}}{\Theta}-\int_{k \Theta}^{E \Theta_{0}} \frac{d t}{t} \frac{1}{\sqrt{1+\left[\frac{2 q}{q^{2}-1}\right]^{2} \frac{\alpha_{s}(t)}{\alpha_{s}(k \Theta)}}} \quad \text { III }-19
$$


While in the fixed coupling regime we recover all the previous results, for example :

$$
\ln \frac{E}{k}=\frac{1}{q^{2}} \ln \frac{\Theta_{0}}{\Theta}\left(=\frac{1}{4} \ln \frac{\Theta_{0}}{\Theta} \text { for } q=2\right) . \quad \text { III }-20
$$

we get now:

$$
\begin{aligned}
& x=\frac{1}{2}\left[1-y\left(1-\int_{y}^{1} \frac{d u}{u^{2}} \frac{1}{\sqrt{1+a_{q} u}}\right)\right], a_{q}=\left(\frac{2 q}{q^{2}-1}\right)^{2} \\
& x=\frac{\ln \Theta_{0} / \Theta}{\ln E \Theta_{0} / \Lambda} ; y=\frac{\ln k \Theta / \Lambda}{\ln E \Theta_{0} / \Lambda}
\end{aligned}
$$

where $\Lambda$ is the QCD scale at first perturbation order.

It is important to notice that the QCD scale $\Lambda$ has replaced the infrared cut-off $\mu$ which appeared in the variable $x_{\mu}$, see [III - 12]. In fact, $\mu$ appears only as the lower bound of the multiplicity contribution to $\mathcal{S}(\omega, k, \Theta)$, see formula [III -15$]$, and disappears from the scaled factorial moments. The final formula will be independent of the infra-red cut-off.

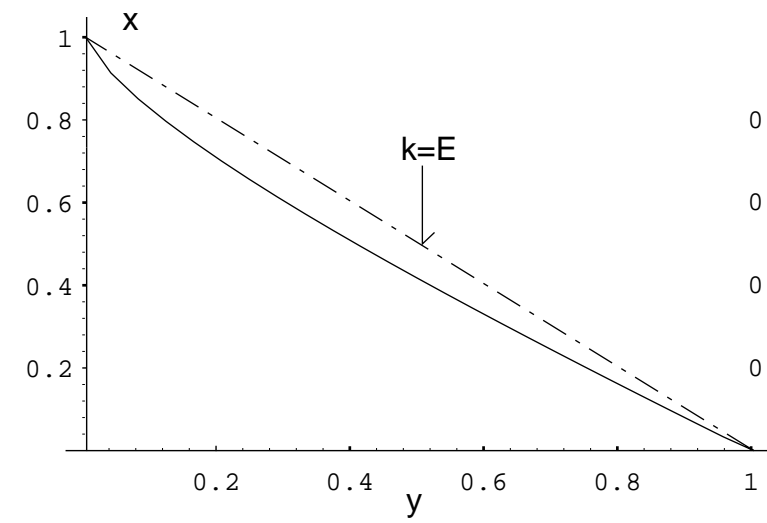

Figure 3a

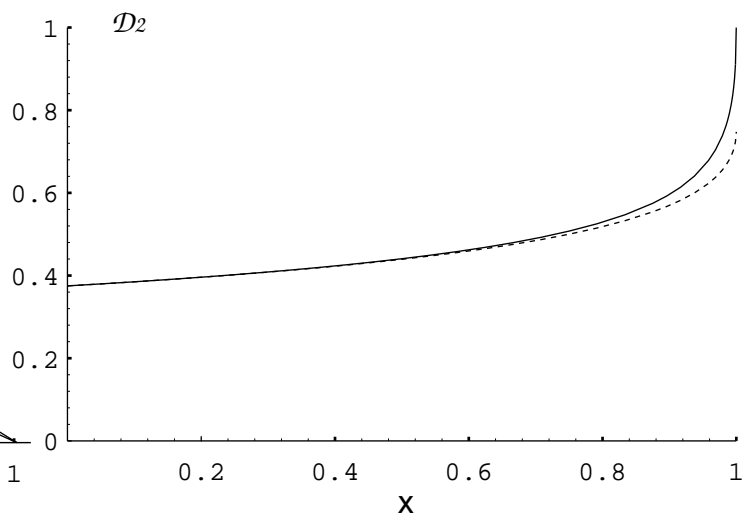

Figure $3 b$

Figure 3: Comparison of angular/momentum hierarchies in a QCD jet fragmentation; a) The scaled-angular $x$ variable as a function of the transverse momentum one $y$. continuous line : The exact formula [ III - 21 ] ; dotted-dashed line : $x=1-y$ corresponding to $k=E$. b) The QCD second intermittency dimension as a function of $\tilde{x}$; continuous line : the exact [ III - 23] formula; dashed curve : the approximate formula [ III - 22 ].

The function $x(y)$ determines how the angular hierarchy in $\ln \Theta_{0} / \Theta$ - measured by its ratio with the maximal value $\ln E \Theta_{0} / \Lambda$ - depends on the transverse 
momentum hierarchy expressed by $1-y=\frac{\ln P_{\perp_{0}} / P_{\perp}}{\ln P_{\perp_{0}} / \Lambda}$. The function $x(y)$ is displayed in figure 3 for $q=2$, together with the function $1-y$, which would correspond to taking $\alpha_{s}(k \Theta)=\alpha_{s}(E \Theta)$. The lesson from Figure 3a is that it is quite consistent to chose $k \simeq E$ in the angular evolution of the running coupling constant $\alpha_{s}(k \Theta)$. The function $x$ can be choosen equal to $1-y$ with a quite good approximation. This leads to the following simple expression fo $\mathcal{D}_{q}$ (cf. also formula [ II -15$]$ )

$$
\begin{aligned}
\mathcal{D}_{q} & =\frac{q+1}{d q} \frac{\int_{\Theta}^{\Theta_{0}} \gamma_{0}\left(\alpha_{s}(E \theta)\right) d \theta / \theta}{\ln \Theta_{0} / \Theta}= \\
& =\frac{q+1}{d q} \gamma_{0}\left(\alpha_{s}\left(E \Theta_{0}\right)\right) \frac{2}{x}(1-\sqrt{1-x}),
\end{aligned}
$$

where $x$ is the same as in [III -21$]$, and $\mathrm{d}$ the dimension. Note again that the result [ III - 22] is independent of the infra-red cut-off.

To be complete, one can work out the exact calculation of the anomalous dimension as a function of $1-y=\tilde{x}$ :

$$
\begin{aligned}
& \mathcal{D}_{q}(\tilde{x})=2 \gamma_{0} \frac{q+1}{d q} \\
& \frac{(1-\sqrt{1-\tilde{x}}) q \sqrt{a_{q}}-a_{q} \sqrt{1-\tilde{x}} \ln \left[q^{2} \frac{1-\sqrt{1+a_{q}(1-\tilde{x})}}{1+\sqrt{1+a_{q}(1-\tilde{x})}}\right]}{1-(1-\tilde{x}) q \sqrt{a_{q}}+\sqrt{1+a_{q}(1-\tilde{x})}+a_{q}(1-\tilde{x}) \ln \left[q^{2} \frac{1-\sqrt{1+a_{q}(1-\tilde{x})}}{1+\sqrt{1+a_{q}(1-\tilde{x})}}\right]} \\
& \simeq 2 \gamma_{0} \frac{q+1}{d q}\left(\frac{1-\sqrt{1-\tilde{x}}}{\tilde{x}}\right)
\end{aligned}
$$

III -23

The exact anomalous dimension $\mathcal{D}_{2}$ is displayed in Figure $3 \mathrm{~b}$ for $d=2$, together with the approximate one, as a function of $\tilde{x}$. The figure shows that the formula [III - 21] (also [ II - 15 ]) is very close to the exact one. This ensures the equivalence between the fluctuation and correlation approaches to the intermittency calculations. In other terms, for factorial moments, the hierarchy in cascading angles is more stringent than the softening in energy. Indeed, both curves differ only slightly in the region $\tilde{x} \simeq 1$ which can be considered outside the validity region for a perturbative calculation, cf. the discussion in the next section IV.

\section{Intermittency properties of a QCD jet}

Let us now examine in a more precise way the intermittency properties of the jet, as given by the final formula [ III -22 ]. We have already noticed that in the case of a fixed running constant, one verifies an exact scaling law [I - 2], where the phase-space cell $\Delta$, in one dimension, is angular. We shall now discuss the physical meaning of the result obtained for the running coupling constant in case of 1 and 2 dimensions. Let us pinpoint a few properties. 
Angular intermittency of $Q C D$ jets

The main feature of formula [III - 22] is that it is expressed in terms of angular variables, namely the diffusion angle $\Theta$ ( in 1 dimension) and the solid angle $\Omega$ (in 2 dimensions). In terms of $\Theta$ in particular, or, more precisely, in terms of the variable $x=\frac{\ln \Theta_{0} / \Theta}{\ln E \Theta_{0} / \Lambda}$, a quite "universal" behaviour is observed, with increasing factorial moments at small $x$, and then saturation and fall near $x=1$ (see Fig. $4 \mathrm{a}$ for 1 dimension, Fig. $4 \mathrm{~b}$ for 2 dimensions). This behaviour can be easily understood from [III - 22]. At small values of $x$ - or not too small cell-sizes $\Theta$ - the intermittent power-law [II - 12,14] is approximatively valid provided one considers the coupling constant $\gamma_{0}$ at $k=E$, i.e. its minimal value within the jet. Then, for smaller cell-size, an interesting saturation effect occurs, which is obtained in the framework of perturbative QCD, independently of any hadronisation model. This saturation effect is entirely due to the running of the coupling constant through the increase of the Renyi dimension with $x$, see [III - 22]. Indeed, this increase becomes so strong that there is an oversaturation effect in the theory, at a value of $\gamma_{0}$ of the order of 1 , which sets the limit of validity of the perturbative expansion of the theory. The physical interpretation is simple, the many gluons produced saturate the fluctuations; indeed, there are so many, that one can no more neglect there reinteractions: We are entering the non perturbative regime.

In Fig. 4b, the result for the two-dimensional case is shown and we remark that the saturation effects are milder; This is a reflexion of the dividing factor $d$ in the Renyi dimension which increases the intermittency strength and thus decreases the relative importance of the saturation effect.

What is the implication of angular intermittency for the usual analysis framework $[1,8-12]$ ? The natural scaling variables emerging from [ III -21$]$ ) are $x$ and $\epsilon$, which are related to angles by:

$$
\ln \Theta_{0} / \Theta=x . \epsilon=x \cdot \ln \left(E \Theta_{0} / \Lambda\right), \quad \text { IV }-1
$$

where the scaling parameter $\epsilon=\ln \left(E \Theta_{0} / \Lambda\right)$ contains all the information on the kinematical parameters of the considered jet evolution (Note that, from Eq. [III - 22], $\mathcal{D}_{q}$ depends on $x$ and on $\gamma_{0}\left(\alpha_{s}\left(E \Theta_{0}\right)\right)$, which itself depends of $\left.\epsilon\right)$. One important consequence is that the value of $\epsilon$ fixes both the energy $(E)$ and the angular $\left(\Theta_{0}\right)$ dependence of the fluctuation patterns. An example of this dependence on $\epsilon$ is given in Fig. 5, for $\mathcal{F}_{2}$ in two dimensions for 2 typical values of $\epsilon$.

Note that the saturation effect is enhanced at smaller $\epsilon$ which means smaller energy and/or smaller angle. We will come back to this interesting property.

Comparison with previous approaches

It is instructive to confront the obtained results - [ III - 22, 23] - and the two different methods we used, fluctuations and correlations, with previous works on the subject ${ }^{[12-17]}$. The formulae [III $\left.-22,23\right]$ and the method leading to them are worth comparing with the results of ref.[15-17]. In ref.[15], the dipole approximation of perturbative QCD cascading led to a determination of the fractal dimension of 


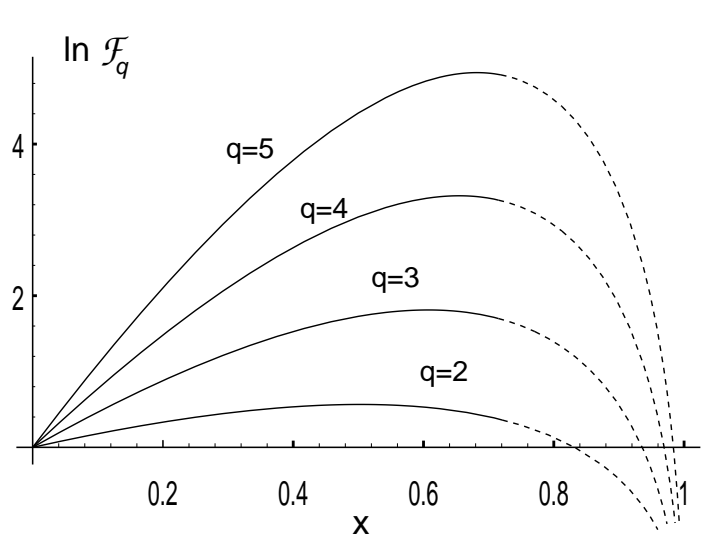

Figure 4a

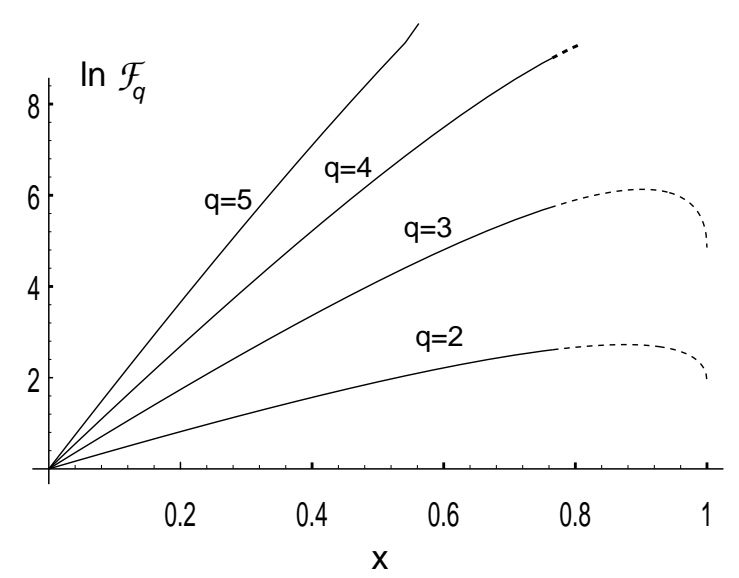

Figure 4b

Figure 4: QCD predictions for the first moments $\mathcal{F}_{q}$ as a function of $x$; a) $d=1, \mathrm{~b}) d=2$. The predictions are computed for the following parameter value : $\epsilon \simeq 5$, which for a jet energy $E \simeq 50 \mathrm{Gev}$ and $\Lambda=200 \mathrm{Mev}$, say, corresponds to an angular aperture of $\Theta_{0} \simeq .6$.

the so-called " $\lambda$ curve" at large values of $q$. This dimension coincide with the large$q$ limit of the approximate Renyi dimension [ III -22 ]. On the other hand, the small- $q$ evolution of the Renyi dimension which characterises the "multi-fractal" feature of QCD gluon cascading is numerically evaluated. Note however, a clear difference in the interpretation of the result: The conclusion of ${ }^{[15]}$ is to propose observables different from factorial moments to exhibit the scale invariant properties of gluon cascading, attributing to hadronisation a dynamical role in the fluctuation pattern. At the level of hadrons, the question is whether gluon-hadron duality is valid or whether hadronisation changes the features of factorial moment observables as suggested in [15]. This deserves more investigation.

We have borrowed from ${ }^{[16]}$ the method using the non-linear equation for multiplicity moments and its transformation in a fragmentation formulation. However, Ref. ${ }^{[16]}$ was only considering planar diagrams with infra-red singular contributions, which are cancelled in the full DLLA (planar + non-planar) diagrams. A comparison with ref.[17] shows that the occurence of a different dominant singularity at small angles, found in ${ }^{[17]}$, is not found in our analysis. Our results are in agreement with those found in ref.[18].

\section{Phenomenological discussion}

Some features present in the Monte-Carlo estimates ${ }^{[11]}$ of the factorial moments based on a Perturbative QCD ansatz are explicit in our analytical solutions, for example the saturation at small cell-size and collimation in azimuth. However, a detailed phenomenological discussion requires an hypothesis on the transformation 


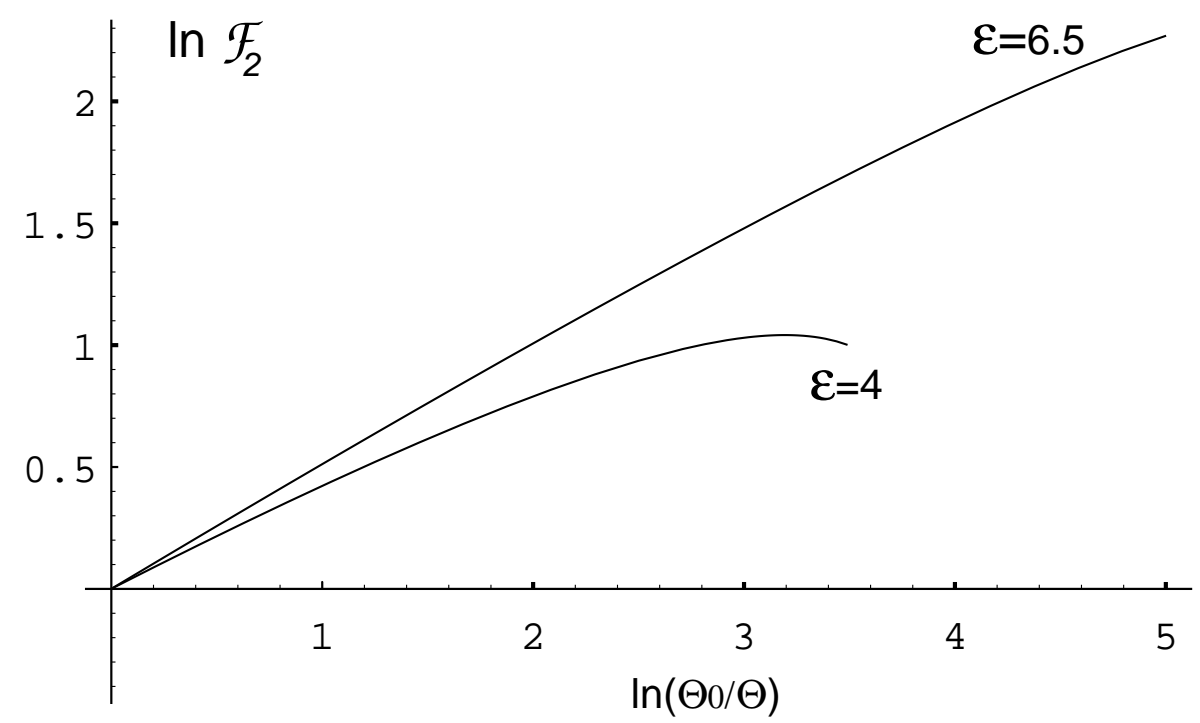

Figure 5

Figure 5 : 2-dimensional $\mathcal{F}_{2}$ as a function of $\ln \Theta_{0} / \Theta$ for two different values of $\epsilon$ (see text).

of partons into hadrons. In fact, we will consider that the effect of hadronisation -which is model-dependent in the Monte-Carlo's- is smooth enough to allow for a direct comparison of hadron experimental data with the predictions of formulae [ III $-22,23]$. This smoothness was already noticed for other observables such as the one-particle inclusive cross-section, the well-known humped-backed plateau in $\mathrm{e}^{+}-\mathrm{e}^{-}$annihilation into hadrons, providing an argument in favor of the LPHD assumption ${ }^{[14]}$. It is interesting to discuss whether or not this property may also apply to multiparticle correlations/fluctuations effects, using factorial moment observables. Note, however, that we did not enter in the computation of next-toleading orders, which are known ${ }^{[19,20]}$ to be important in 2-particle or higher order correlations. Indeed, DLLA partonic evaluation seems to overestimates the correlations in a systematic way. We thus have to find predictions which would minimize the higher-order and hadronisation corrections.

The experimental data on factorial moments and intermittency in $\mathrm{e}^{+}-\mathrm{e}^{-}$annihilation into hadrons and especially on hadronic decays of $\mathcal{Z}_{0}$ 's are already remarkable ${ }^{[10,11]}$. A few features which we discussed previously, like the saturation effects, the increase of intermittency with dimensionality, the dependence of the diffusion direction angle can be seen in the data. However, the tests of angular intermittency need a reappraisal of the most convenient observables. Indeed, no data exist directly in terms of the $\Theta$ variables, and it would be unfair to make uncontrolled 
combinations of the existing data ${ }^{[11]}$ depending on rapidity. We thus propose new observables in order to check the QCD predictions.

The best indication for confronting angular intermittency with data - if experimentally feasible - would be to give one- and two-dimensional factorial moments directly for small cells in angular variables. Indeed, let us consider a given direction $\Theta_{0}$, and two angular cells of aperture $\Theta_{1}$ and $\Theta_{2}$ around that direction. Using twice [II -15$]$, one gets:

$$
\mathcal{F}_{q}\left(\Theta_{1}\right)=\mathcal{F}_{q}\left(\Theta_{2}\right) \exp \left[(q-1)\left(\log \Theta_{2} / \Theta_{1}-\frac{q+1}{q} \int_{\Theta_{1}}^{\Theta_{2}} \gamma_{0}\left(\alpha_{s}(E \theta)\right) d \theta / \theta\right)\right]
$$

$[\mathrm{IV}-2]$ is formally independent of the chosen direction $\Theta_{0}$, except that one has to take into account the application range of perturbative calculations, namely:

$$
0<\log \frac{\mu}{\Lambda}<\log \frac{E \Theta_{1}}{\Lambda}<\log \frac{E \Theta_{2}}{\Lambda}<\log \frac{E \Theta_{0}}{\Lambda}=\epsilon \quad \text { IV }-3
$$

For example, in Fig. 6, we display the predictions for $\mathcal{Z}_{0}$ jet decays corresponding to 1-dimensional ( $\varphi$-averaged) and 2-dimensional moments of rank 3 as a function of $\log \Theta_{2} / \Theta_{1}$ for two typical values of $\epsilon_{2}=\log E \Theta_{2} / \Lambda$. Following [ IV -3 ], the range of the variable $\log \Theta_{2} / \Theta_{1}$ between 0 and $\log E \Theta_{0} / \mu \simeq 3$.

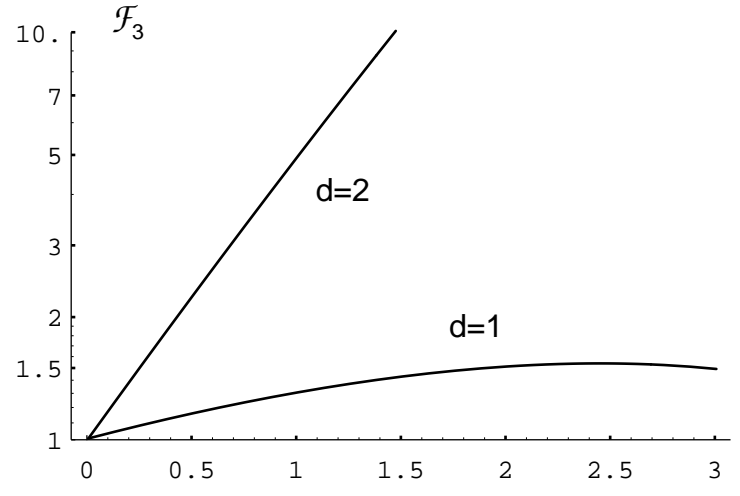

Figure 6a

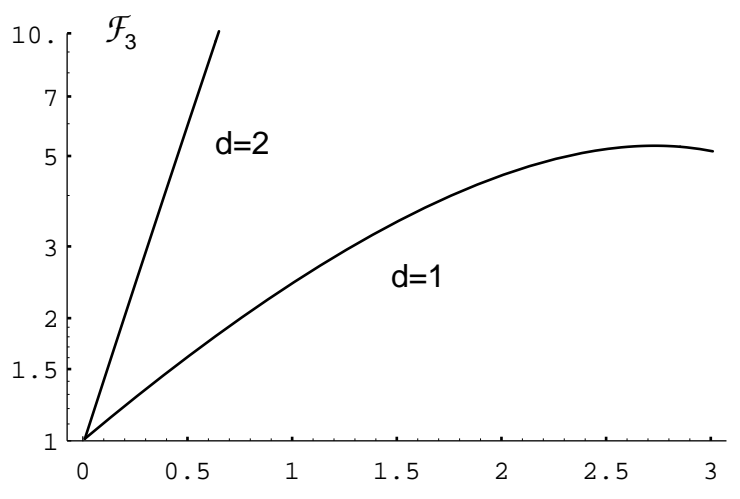

Figure $6 b$

Figure $6: \mathcal{F}_{3}$ ratios as a function of $\Theta_{2} / \Theta_{1}$ (in $\log / \log$ units); a) $\epsilon_{2}=6$; b) $\epsilon_{2}=4$.

Note that the saturation effect, clearly present in the one dimensional case, almost desappears for 2 - $\mathrm{d}$ solid angles cells. Data on factorial moments are in general 
(except those from ALEPH collaboration) "horizontally" averaged over different rapidities - i.e. different $\Theta_{0}$ angles -. We suggest to perform ratios of moments for different apertures before an eventual horizontal averaging in order to test the prediction $[I V-2]$. It is interesting to notice that, if this ratio can be measured event by event, the result is predicted to be independent from the angular direction $\Theta_{0}$, and thus from the definition of the jet axis! However, one has to require to avoid small angles $\Theta_{0}$, (a non-perturbative region for QCD) and thus the determination of jets cannot be totally ignored. In any case, the check of [IV -2$]$ represents a challenge for QCD phenomenology, taking into account that higher-order and hadronisation corrections are probably diminished in the ratio of moments .

\section{Conclusions and outlooks}

The conclusions of our study can be cast in the form of a summary of answers to the questions $i-v$ ) raised in the introduction;

i) Perturbative QCD is intrinsically intermittent, at least in the Double Leading Logarithm Approximation (DLLA), since the factorial moments develop an (effective) singular behaviour in small phase-space cells away from the jet axis, see [ II -15 , III -22 ]. However, instead of a singularity in rapidity cell-size, as in previous studies, one obtains a singularity in angular cell-size.

ii) Intermittent patterns of fluctuations appear in angular variables, namely diffusion and azimuthal angles around the jet axis, and solid angle for 2-dimensional fluctuations. The resulting angular intermittency manifests itself as the enhancement of gluon multiplicity fluctuations in smaller and smaller angular cells in a given direction away from the initial jet axis. Non-perturbative or higher-order corrections invalidate the calculation for too small cells or angles with respect to the jet axis.

iii) The intermittency indices, characterizing the singular behaviour of fluctuations, are related to the QCD anomalous dimension of multiplicities $\gamma_{0}$, and through it, depend only on the scaling parameter $\epsilon=\log E \Theta / \Lambda$, where $\Theta$ is the angular cell-size.

iv) Angular intermittency predicts that the ratio of factorial moments for two cells of different size around the same direction depends only and specifically on these two sizes. We suggest to use angular variables in the phenomenological analysis of $Z_{0}$ decays into hadrons to check this prediction, assuming parton-hadron duality.

$v$ ) The reason why the intermittency singularity appears is primarily due to the resummation of the infra-red potential divergencies of the theory through their leading-log contributions at all orders; The size of the angular cell, over which one integrates to get factorial moments, acts as a regulator scale over these potential infra-red divergencies.

Questions to be solved in the near future are not lacking, starting with the abovementionned suggestions and requirements for experimental studies. Among 
other problems, it seems that the 3 - $d$ fluctuations, when one adds transverse momentum in the game, are at hand. The study of the transition to the nonperturbative region and its fluctuation properties could be facilitated by its kinematical location in the fragmentation rapidity domain. A related question is to ask whether other multi-particle reactions present similar features as $\mathrm{e}^{+}-\mathrm{e}^{-}$. This would give an hint on possible mixing of perturbative and other dynamical fluctuation mechanisms; One would of course think to deep-inelastic reactions at HERA, but also to hadron or nuclei-induced reactions.

As a final and more speculative remark, it is tempting to consider the QCD calculations as a new laboratory for the unsolved problem of turbulence. It is

quite clear that turbulence is by far a more complex problem, but the existence of a gauge field theory like QCD supporting the existence of self-similar fluctuation patterns and experiments of high quality to discuss it could give some help. After all, long ago, the possibility of a fractal behaviour of jets into jets into jets... has been beautifully predicted ${ }^{[25]}$. It is quite remarkable that the property of angular intermittency in QCD jets seems to realize this prediction in a quite non-trivial way since there appears a $q$-dependent and angular-dependent set of fractal dimensions (multi-fractality property).

\section{Acknowledgments}

We warmly thank Yuri Dokshitzer for stimulating discussions which convinced us to use for the present work one basic (correlation) method developed by him and coworkers ; We acknowledge him for helpful remarks and communicating related unpublished notes (with Serguei Troyan). We had also fruitful discussions with Bo Andersson, Andrzej Bialas, Wolfgang Ochs and Jacek Wosiek at the occasion of recent meetings and conferences (Orsay/Saclay, Santiago, Lisbon,...). We thank Henri Navelet for final remarks on the draft. 


\section{References}

1 For a review and early references, see A. de Angelis, P. Lipa and W. Ochs, Proceedings of the Joint LP-HEP Conference 91, Vol. 1, page 724 (Eds. S. Hegarty, K. Potter, E. Quercigh, World Scientific).

2 A. Białas and R. Peschanski, Nucl. Phys. B 273 (1986) 703, 308 (1988) 857.

3 For general review on the subject: A. Białas Nucl. Phys. A525 345c (1991); R. Peschanski, Int. J. Mod. Phys.A6 (1991) 3681.

4 P. Carruthers and I. Sarcevic, Phys. Rev. Lett. 63 (1989) 1562, and refs. in $[3]$.

5 P. Lipa and B. Buschbeck, Phys. Lett.B223 (1989) 465 and references therein.

6 P. Carruthers, J. Stat. Phys.51 (1988) 517.

7 A. Białas and J. Seixas, Phys. Lett.B250 (1990) 151.

8 For a recent experimental review, see F. Verbeure, Proceedings of the XXII International Symposium on Multiparticle Dynamics, Santiago de Compostela, 13-17 July 1992 (to be published soon, World Scientific).

9 See, for instance, refs $[1,3]$ and various contributions to the proceedings of ref. [8].

10 B. Buschbeck, P. Lipa and R. Peschanski, Phys. Lett.B215 (1988) 788; HRS coll., TASSO coll., CELLO coll. references in [1].

11 Last LEP results: DELPHI Coll., P. Abreu et al. Nucl. Phys.B386 (1992) 471; ALEPH Coll., D. Decamp et al. Z. Phys.C53 (1992) 21; See previous references (including OPAL and L3 published results, 1991) therein.

12 M. Jedrejczak, Phys. Lett. B 228 (1988) 259.

13 C.B. Chiu and R.C. Hwa, Phys. Lett. B 236 (1990) 446.

14 Y.I. Azimov, Y.L. Dokshitzer, V.A. Khoze, and S.I. Troyan, Z. Phys.C 31 (1986) 213.

15 G. Gustafsson and A. Nilsson, Z. Phys. C 52 (1991) 533;

16 Ph. Brax and R. Peschanski, Saclay Preprint SphT/92-005(1992) .

17 W. Ochs and J. Wosiek, Phys. Lett. B289 (1992) 159.

18 Y.L. Dokshitzer and S.I. Troyan, unpublished (1991).

19 Y.L. Dokshitzer, G. Marchesini and G. Oriani, Nuclear Physics B 387 (1992) 675.

20 For a review, see for example Basics of Perturbative QCD Y.L. Dokshitzer, V.A. Khoze, A.H. Mueller and S.I. Troyan (J. Tran Than Van ed., Editions Frontieres) 1991, and the list of references therein.

21 Note that the equation [ II - 2] is valid beyond DLLA, see ref. [18], provided one chooses a running QCD scale $\alpha_{s}\left(P_{\perp}\right)$, where $P_{\perp} \simeq k \Theta_{0}, k$ being the current parton energy with an infrared cut-off $\mu$; The condition : $\mathcal{Z}\left(P_{\perp} \equiv \mu, v\right)=v$ has to be fulfiled. 
22 J.-L. Meunier and R. Peschanski, Nuclear Physics B 374 (1992) 327,

23 Y. Gabellini, J.-L. Meunier and R. Peschanski, Z. F. Phys. C 55 (1992) 455.

24 W. Ochs, Z. Phys.C 50 (1991) 379; Act. Phys. Pol.B 25 (1991) 203. A. Bialas and M. Gazdzicki, Phys. Lett.B 252 (1990) 483.

25 G. Veneziano, Proc. 3rd. Workshop on Current Problems in HEP theory, Florence 1979, eds. Casalbuoni et al; John Hopkins University Press, Baltimore. 


\section{Figure captions}

Figure 1 : Sketch of the random fragmentation cascade. In grey, the "history" of the bin $\Delta$, i.e. the successive random multiplicative factors $\mathrm{w}$ which define the multiplicity density in $\Delta$ for one event. $Y$ (resp. $\Delta)$ is the initial (resp. final) observation range. As a simple illustration from the figure, the bin density is $\rho_{\Delta}=\rho_{Y} \mathrm{w}_{1} \mathrm{w}_{2} \mathrm{w}_{3} \mathrm{w}_{4}$ where $\rho_{Y}$ is the initial density, and the w's are the random or unity weights (see text) corresponding to the semi-random structure.

Figure 2 : Kinematics of parton-parton QCD correlations; a) Location of the phase-space cell $\Delta$ in polar coordinates; b) Schematic representation of the convolution formulae [ III - 1,5].

Figure 3: Comparison of angular/momentum hierarchies in a QCD jet fragmentation; a) The scaled-angular $x$ variable as a function of the transverse momentum one $y$. Continuous line : The exact formula [ III - 21 ] ; dotted-dashed line : $x=1-y$ corresponding to $k=E$. b) The QCD second intermittency dimension as a function of $\tilde{x}$; continuous line : the exact [ III - 23] formula; dashed curve : the approximate formula [ III - 22 ].

Figure 4: QCD predictions for the first moments $\mathcal{F}_{q}$ as a function of $x$; a) $d=1, \mathrm{~b}) d=2$. The predictions are computed for the following parameter value : $\epsilon \simeq 5$, which for a jet energy $E \simeq 50 G e v$ and $\Lambda=200 \mathrm{Mev}$, say, corresponds to an angular aperture of $\Theta_{0} \simeq 6$.

Figure 5 : 2-dimensional $\mathcal{F}_{2}$ as a function of $\ln \Theta_{0} / \Theta$ for two different values of $\epsilon$ (see text).

Figure $6: \mathcal{F}_{3}$ ratios as a function of $\Theta_{2} / \Theta_{1}($ in $\log / \log$ units $\left.\left.) ; a\right) \epsilon_{2}=6 ; \mathrm{b}\right)$ $\epsilon_{2}=4$. 